\title{
Infected hepatic hematoma 10 days after ERCP
}

An 84-year-old man with severe (GOLDIV) chronic obstructive pulmonary disease (COPD) and a history of pulmonary thromboembolism and warfarin medication was admitted for elective endoscopic retrograde cholangiopancreatography (ERCP) for choledocholithiasis. He had stopped warfarin medication a week before (international normalized ratio [INR] 1.14), and was kept on low-molecular-weight heparin.

ERCP was performed and guidewire cannulation of the common bile duct was achieved easily ( Fig. 1). Sphincterotomy was not necessary. Balloon extraction was accomplished uneventfully. The patient restarted warfarin medication but was not discharged due to exacerbated COPD. After 10 days he developed an intense right upper quadrant abdominal pain accompanied by a hemoglobin drop (14 to $10.7 \mathrm{~g} / \mathrm{dL}$ ) and INR elevation (4.22). Abdominal ultrasound/computed tomography scan revealed a heterogeneous mass on the right liver lobe measuring $9 \times 10 \mathrm{~cm}$ ( Fig.2). Anticoagulation was suspended.

After 3 days, sepsis was apparent, with fever, leukocytosis $\left(23880 / \mathrm{mm}^{3}\right)$, and elevated C-reactive protein $(27 \mathrm{mg} / \mathrm{dL})$. The hematoma was punctured and treatment with meropenem was started; vancomycin was added to the treatment regimen after the isolation of Enterococcus faecium.

Response to treatment was favorable and antibiotic treatment was continued for 4 weeks, but sepsis returned soon after antibiotic treatment had finished ( $\bullet$ Fig.3). Percutaneous drainage was performed ( $\bullet$ Fig.4). The patient stayed in hospital for 5 months due to recurrent infection of the liver lesion and episodes of COPD and pneumonia. He died after 5 months.

Subcapsular hematoma following ERCP is a complication that was first described in 2000 [1], and a few other cases have been reported subsequently [2-5]. It is not uncommon for patients to be discharged from hospital only to be re-admitted later the same day or up to 5 days later [5]. In one case, infection was managed with percutaneous drainage and antibiotic therapy [1].

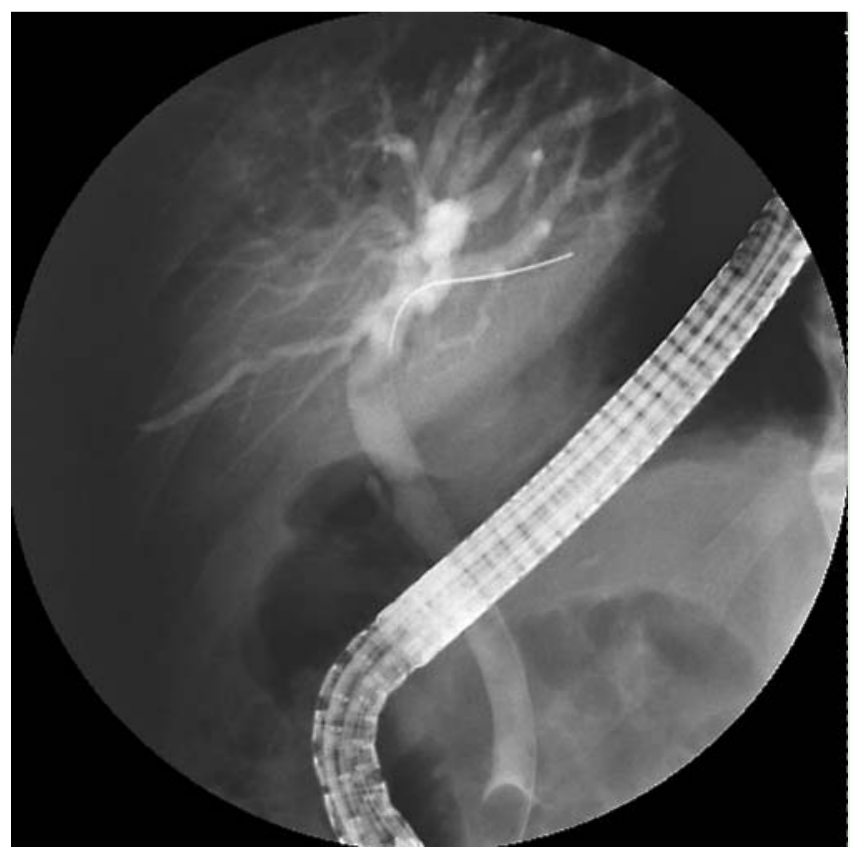

Fig. 1 Endoscopic retrograde cholangiopancreatography cholangiogram after cannulation with 0.035 inch straight tip Jagwire (Boston Scientific Corporation, Natick, Massachusetts, USA).

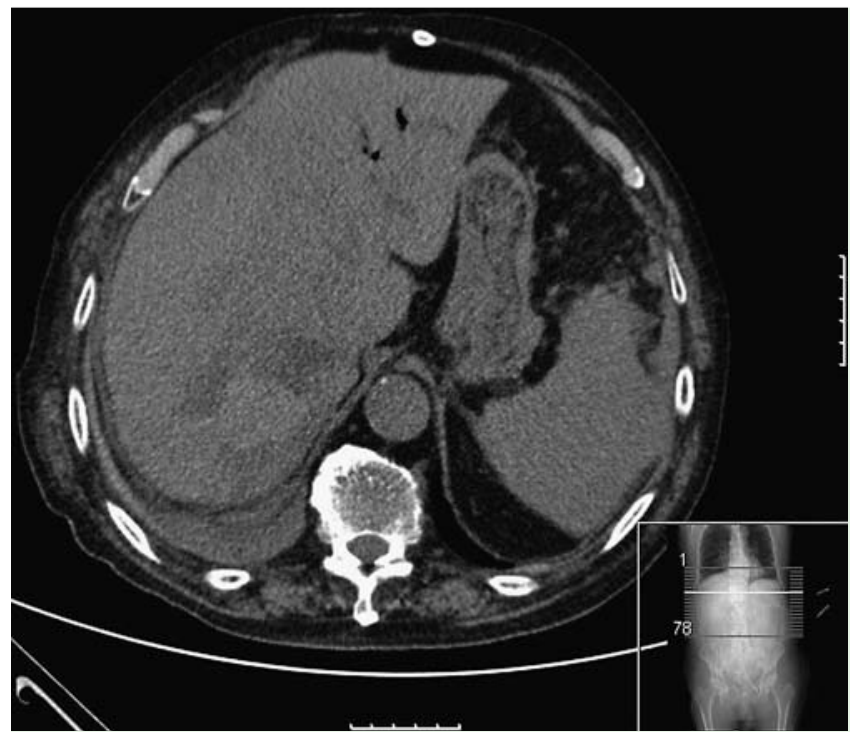

Fig. 2 Abdominal computed tomography scan at Day 13 showing heterogeneous hypodensity on the right liver lobe.

Although hepatic hematoma is considered to be a rare complication of ERCP, the incidence may be higher than thought, due to an increase in the use of the new oral anticoagulants. It may develop even in the absence of sphincterotomy and the risk is probably increased in the hypocoagulation setting, demanding strict INR control for at least 10 days after the procedure.
Endoscopy_UCTN_Code_CPL_1AK_2AI

Competing interests: None

\section{Alexandre Oliveira Ferreira ${ }^{1,2}$, Rui Tato Marinho², José Velosa ${ }^{2}$}

${ }^{1}$ Department of Gastroenterology, Unidade de Portimão - Centro Hospitalar do Algarve, Portimão, Portugal

2 Department of Gastroenterology and Hepatology, Medical School of Lisbon, Hospital Santa Maria, Lisbon, Portugal 



Fig. 3 Intravenous contrast-enhanced abdominal computed tomography scan 2 months after endoscopic retrograde cholangiopancreatography, showing free gas in the hypodense lesion as a result of infection and bacteria metabolism.

\section{References}

1 Ortega Deballon P, Fernández Lobato R, García Septiem J et al. Liver hematoma following endoscopic retrograde cholangiopancreatography (ERCP). Surg Endosc 2000; 14: 767

2 Chi KD, Waxman I. Subcapsular hepatic hematoma after guidewire injury during endoscopic retrograde cholangiopancreatography: management and review. Endoscopy 2004; 36: 1019-1021

3 Bhati CS, Inston N, Wigmore SJ. Subcapsular intrahepatic hematoma: an unusual complication of ERCP. Endoscopy 2007; 39 (Suppl. 01): E150

4 Orellana F, Irarrazaval J, Galindo J et al. Subcapsular hepatic hematoma post ERCP: a rare or an underdiagnosed complication? Endoscopy 2012; 44 (Suppl. 02): E108 - 109

5 Del Pozo D, Moral I, Poves E et al. Subcapsular hepatic hematoma following ERCP: case report and review. Endoscopy 2011; 43 (Suppl. 02): E164-165

Fig. 4 Intravenous contrast-enhanced abdominal computed tomography scan 2 months after endoscopic retrograde cholangiopancreatography with the percutaneous drainage catheter visible. 